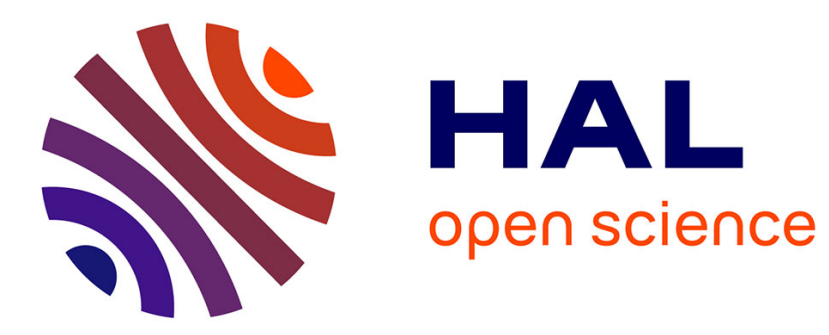

\title{
Topological segmentation of discrete surfaces
}

\author{
Grégoire Malandain, Gilles Bertrand, Nicholas Ayache
}

\section{To cite this version:}

Grégoire Malandain, Gilles Bertrand, Nicholas Ayache. Topological segmentation of discrete surfaces. International Journal of Computer Vision, 1993, 10 (2), pp.183-197. 10.1007/BF01420736 . inria00615046

\section{HAL Id: inria-00615046 \\ https://hal.inria.fr/inria-00615046}

Submitted on 17 Aug 2011

HAL is a multi-disciplinary open access archive for the deposit and dissemination of scientific research documents, whether they are published or not. The documents may come from teaching and research institutions in France or abroad, or from public or private research centers.
L'archive ouverte pluridisciplinaire HAL, est destinée au dépôt et à la diffusion de documents scientifiques de niveau recherche, publiés ou non, émanant des établissements d'enseignement et de recherche français ou étrangers, des laboratoires publics ou privés. 
International Journal of Computer Vision, 10:2, 183-197 (1993)

(C) 1993 Kluwer Academic Publishers, Manufactured in The Netherlands.

\title{
Topological Segmentation of Discrete Surfaces
}

\author{
GRÉGOIRE MALANDAIN \\ INRIA-Domaine de Voluceau-Rocquencourt-78153 Le Chesnay Cédex, France
}

GILLES BERTRAND

ESIEE-Labo IAAI-Cité Descartes-2, boulevard Blaise Pascal-93162 Noisy-le-Grand Cédex, France

NICHOLAS AYACHE

INRIA-Domaine de Voluceau-Rocquencourt-78153 Le Chesnay Cédex, France

Received July 16, 1992. Revised July 27 and November 4, 1992.

\begin{abstract}
This article proposes a new approach to segment a discrete 3-D object into a structure of characteristic topological primitives with attached qualitative features. This structure can be seen itself as a qualitative description of the object, because

- it is intrinsic to the 3-D object, which means it is stable to rigid transformations (rotations and translations);

- it is locally defined, and therefore stable to partial occlusions and local modifications of the object structure;

- it is robust to noise and small deformations, as confirmed by our experimental results.

Our approach concentrates on topological properties of discrete surfaces. These surfaces may correspond to the external surface of the objects extracted by a 3-D edge detector, or to the skeleton surface obtained by a new thinning algorithm. Our labeling algorithm is based on very local computations, allowing massively parallel computations and real-time computations.

An indirect result of these topological properties is a new characterization of simple points.

We present a realistic experiment to characterize and locate spatially a complex 3-D medical object using the proposed segmentation of its skeleton.
\end{abstract}

\section{Introduction}

Three-dimensional (3-D) images may come from several fields, the most popular one being the medical field, where images are produced by X-ray Computed Tomography (CT), Magnetic Resonance Imaging (MRI) (see figure 1), Positon Emitting Tomography (PET), and more recently by Ultrasound Echography. Automating the interpretation of these images is an awkward but important task for many applications where extremely accurate quantitative results are required or/and a large volume of data must be processed. A large class of interpretation tasks involves a matching stage, an accurate geometric registration between two 3-D images, or between a 3-D image and a 3-D geometric and semantic model (Ayache et al. 1990).
Prior to matching, a preliminary segmentation stage is necessary to reduce the original image into a more compact highly structured representation useful for interpretation (Ayache et al. 1989).

Here, we propose a new segmentation process which transforms a discrete binary 3-D object into a structure of characteristic topological primitives (such as volumes, surfaces, curves, etc.) with attached qualitative features. This structure can be seen itself as a qualitative description of the object, because

- it is intrinsic to the 3-D object, which means it is stable to rigid transformations (rotations and translations);

- it is locally defined, and therefore stable to partial occlusions and local modifications of the object structure; 
- it is robust to noise and small deformations, as confirmed by our experimental results.

The idea of characterizing 3-D shapes with qualitative primitives was introduced by Sander (1989) who studied differential singularities of 3-D object surfaces.

Our approach is complementary and original because we concentrate on different topological properties of discrete surfaces instead of geometrical properties. Finally, the core of our approach is based on very local computations, allowing massively parallel computations and real-time computations.

Let us consider a 3-D image (like in figure 1). We can segment it by using classical algorithms of edge detection (Monga 1990) (see figure 2). These edges are surfaces in 3-D. In figure 3 we have the same object (made of surfaces) in two different positions. Using the proposed topological segmentation, we will be able to extract these surfaces and their junctions, and therefore to have a structured description of them in terms of surfaces and junctions. This description will be a precious help for any further stage (like matching), An indirect result of this topological segmentation is a new characterization of 3-D simple points, which leads to a new thinning algorithm.

This article is organized as follows. First we present the concept of topological segmentation. Second, we recall previous work done on digital topology. Third, we describe our approach which is twofold, (a) local labeling of object points using a classification tree with two local measures, and (b) detection of junctions
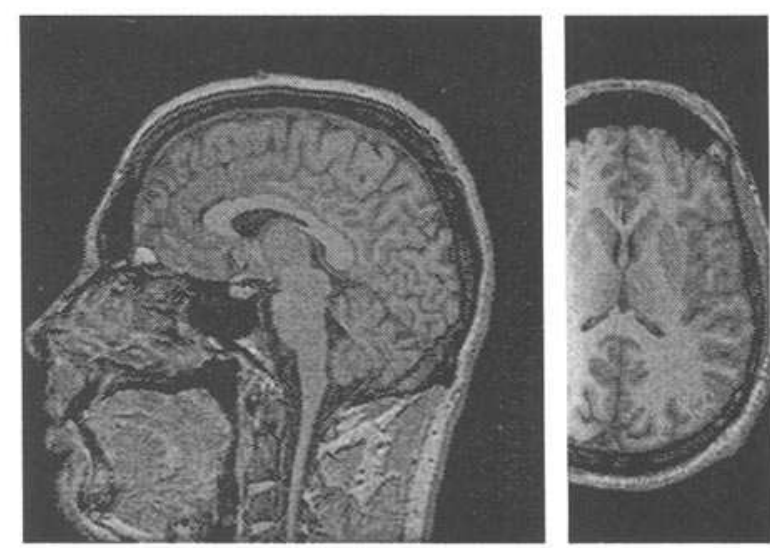

Fig. 1. Two sections of a 3-D MRI image of a head.
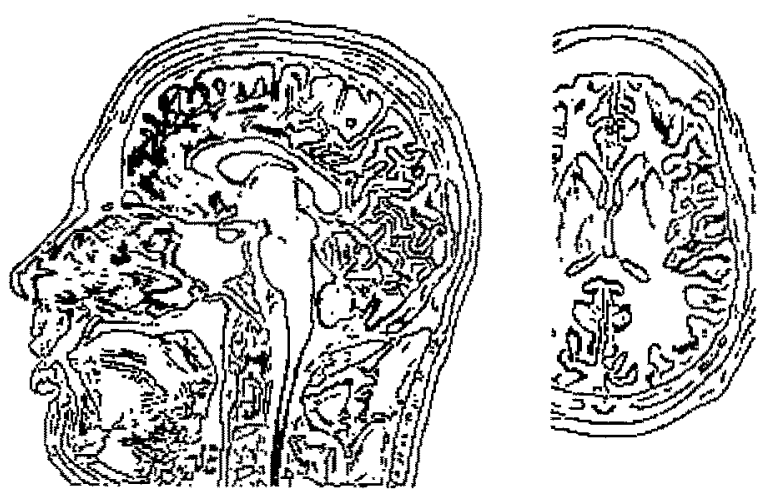

Fig. 2. Two sections of edges detected in the 3-D MRI image of figure 1 .
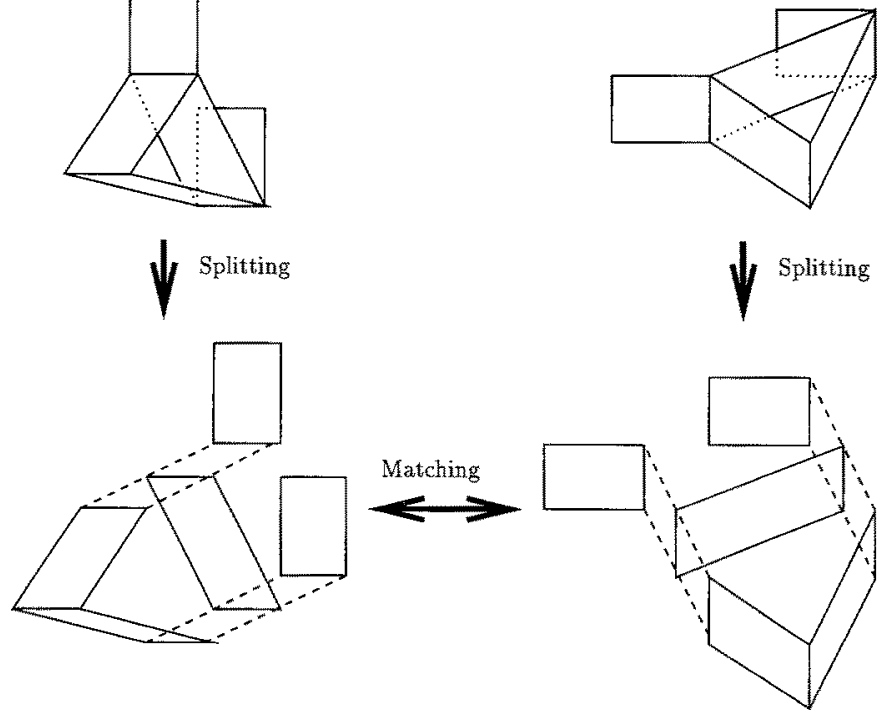

Fig. 3. Topological segmentation gives us a description of each object, by splitting it into simple surfaces. This description can be useful for a later step of matching. 
using a new characterization of simple surfaces. Fourth, after discussing the need for a thinning preprocessing before the topological segmentation, we propose a new characterization of simple points derived from our classification and a new thinning algorithm. Fifth, we propose the use of mathematical morphology to refine the detection of simple surfaces. Sixth, we describe two applications of this work, one using our thinning algorithm and the other using our classification for the segmentation and the spatial localization of the skeleton of a cómplex 3-D medical object. We conclude with a study of the advantages of this approach and some short and medium term future research topics.

\section{Topological Classification in $\mathbf{I R}^{3}$}

Before giving an introduction to digital topology, we first present the concept of topological segmentation in $\mathbb{R}^{3}$. This will help the reader to grasp the ideas developed in sections 3 and 4 . We have a binary partition of the real space in two parts: the object $X$ and the background $\bar{X}$. An illustration of such an object is shown in figure 4 . The goal of the topological classification is to characterize each point of a binary 3-D image by its topological type.

Each point may belong to one of the following categories:

volume: one can find a neighborhood of the point that contains no point of the background.

surface: one can find a neighborhood of the point such that the background is divided in two parts by the object. curve: one can find a neighborhood of the point such that the deletion of the point involves the division of the object in two parts.

isolated point: one can find a neighborhood of the point such that the deletion of the point involves the deletion of the object.

Or a point may be a combination between two or more of these categories (for example, it may be a junction point between a curve and a surface). Note that a point may also be a border point (a border point is a point that can be suppressed without changing the topology of the object).

Each category is self-defined and is simply characterized by the number of connected components in a neighborhood (connected components of the background for volumes and surfaces and of the object for curves and isolated points).

Let us consider a point $x$ of the object $X$, and a neighborhood $V$ of $x$ (in a topological sense: $V$ contains an open set which contains $x$ ). Let $V^{*}=V \backslash\{x\}$ be the same neighborhood without the point $x$. We can compute four numbers of connected components, two for the object and two for the background by considering the point $x$ either in the object or in the background. We denote these four numbers by:

$C$ number of connected components of the object in $V$

$C^{*}$ number of connected components of the object in $V^{*}$

$\bar{C}$ number of connected components of the background in $V$

$\bar{C}^{*}$ number of connected components of the background in $V^{*} \cup\{x\}$ ( $x$ is considered as a point of the background)

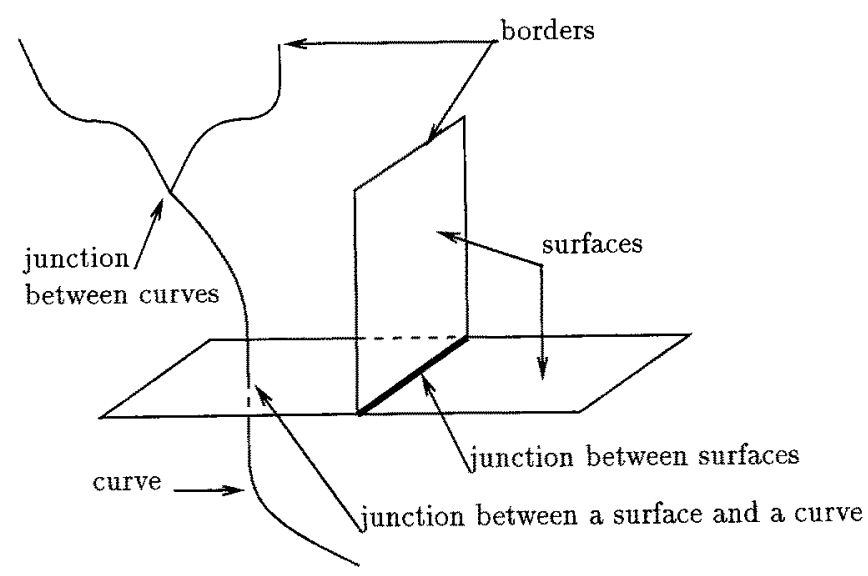

Fig. 4. Different topological types. 
These numbers depend on the choice of the neighborhood $V$. This choice must then satisfy some properties.

In order to count connected components, we choose a neighborhood $V$ that verifies the following:

Property 1. $V$ must be connected (and then $V^{*}$ is connected).

Property 2. For any connected neighborhood $W$ of $x$ included in $V$, the values of $C, C^{*}, \bar{C}$, and $\bar{C}^{*}$ are the same.

If the object is "topologically regular enough" (and so are all the objects we usually have in a digital space), we can find such a neighborhood for any point.

We can easily prove that, in such a neighborhood, we have:

Proposition 1. In a neighborhood $V$ that satisfies properties 1 and 2 , we have

$$
C=\bar{C}^{*}=1
$$

Then, only two numbers are necessary for the topological classification, $C^{*}$ and $\bar{C}$. $C^{*}$ is used for characterizing isolated points and curves.

$$
\begin{aligned}
& C^{*}=0 \begin{array}{l}
\text { there is no connected component } \\
\text { of the object in } V^{*}, x \text { is isolated. }
\end{array} \\
& C^{*}=1 \quad \begin{array}{l}
x \text { is neither an isolated point nor } \\
\text { a curve point. }
\end{array} \\
& C^{*}=2 \begin{array}{l}
\text { locally, the deletion of } x \text { divides } \\
\text { the object in two parts; } x \text { belongs } \\
\text { to a curve. }
\end{array} \\
& C^{*} \geq 3 \begin{array}{l}
\text { locally, the deletion of } x \text { divides the } \\
\text { object in three or more parts; } x \text { be- } \\
\text { longs to a junction between curves. }
\end{array}
\end{aligned}
$$

and $\bar{C}$ is used for characterizing surfaces and volumes:

$$
\begin{aligned}
& \bar{C}=0 \begin{array}{l}
\text { there is no connected component } \\
\text { of the background in } V, x \text { is an in- } \\
\text { terior point of a volume. }
\end{array} \\
& \bar{C}=1 \quad \begin{array}{l}
x \text { belongs neither to a volume, nor } \\
\text { a surface. }
\end{array} \\
& \bar{C}=2 \begin{array}{l}
\text { locally, the background is divided } \\
\text { in two parts; } x \text { belongs to a surface. }
\end{array} \\
& \bar{C} \geq 3 \begin{array}{l}
\text { locally, the background is divided } \\
\text { in three or more parts, } x \text { belongs } \\
\text { to a junction between surfaces. }
\end{array}
\end{aligned}
$$

We can thus propose a topological classification for each point of a binary partition of $\mathbb{R}^{3}$ using the two numbers $C^{*}$ and $\bar{C}$ (see table 1 ).

Table 1. Toplogical classification of a point using the two numbers $C^{*}$ and $\bar{C}$.

\begin{tabular}{llll}
\hline Type A & interior point & $\bar{C}=0$, & \\
Type B & isolated point: & $\bar{C}=1, C^{*}=1$ \\
Type C & border point: & $\bar{C}=1, C^{*}=2$ \\
Type D & curve point: & $\bar{C}=1, C^{*}>2$ \\
Type E & curves junction: & $\bar{C}=2, C^{*}=1$ \\
Type F & surface point: & $\bar{C}=2, C^{*} \geq 2$ \\
Type G & surface-curve(s) junction: & $\bar{C}>2, C^{*}=1$ \\
Type H & surfaces junction: & $\bar{C}>2, C^{*} \geq 2$ \\
Type I & surfaces-curve(s) junction: & \\
\hline
\end{tabular}

\section{Digital Topology}

We present some basic definitions of 3-D digital topology-see (Rosenfeld 1980; Kong \& Rosenfeld 1989; Nakamura \& Aizawa 1985; Toriwaki et al. 1982).

\subsection{Neighborhoods and Connectivities}

A 3-D digital image $\Sigma$ is a subset of $\mathbb{Z}^{3}$. We consider only cubic lattices so that, for each point $x=(i, j, k)$, three types of neighbors may be defined:

6-neighbors: two points are 6-neighbors if they share a face.

18-neighbors: two points are 18-neighbors if they share a face or a vertex.

26-neighbors: two points are 26-neighbors if they share either a face, or a vertex, or a corner.

Each of these three types defines a connectivity denoted by $n$-connectivity (for $n \in\{6,18,26\}$ ). We have then three different neighborhoods of a point $x$ (see figure 5) denoted by $N_{n}(x)$. We note $N_{n}^{*}(x)=$ $N_{n}(x) \backslash\{x\}$. Let us remark that 6-connectivity and 26-connectivity correspond to two distances of $\mathbb{R}^{3}$ often denoted $D_{1}$ and $D_{\infty}$.

Let $x=\left(x_{1}, x_{2}, x_{3}\right)$ and $y=\left(y_{1}, y_{2}, y_{3}\right)$ be two points, these two metrics are defined by

$$
\begin{aligned}
& D_{1}(x, y)=\sum_{i=1, \ldots, 3}\left|y_{i}-x_{i}\right| \\
& D_{\infty}(x, y)=\max _{i=1, \ldots, 3}\left|y_{i}-x_{i}\right|
\end{aligned}
$$



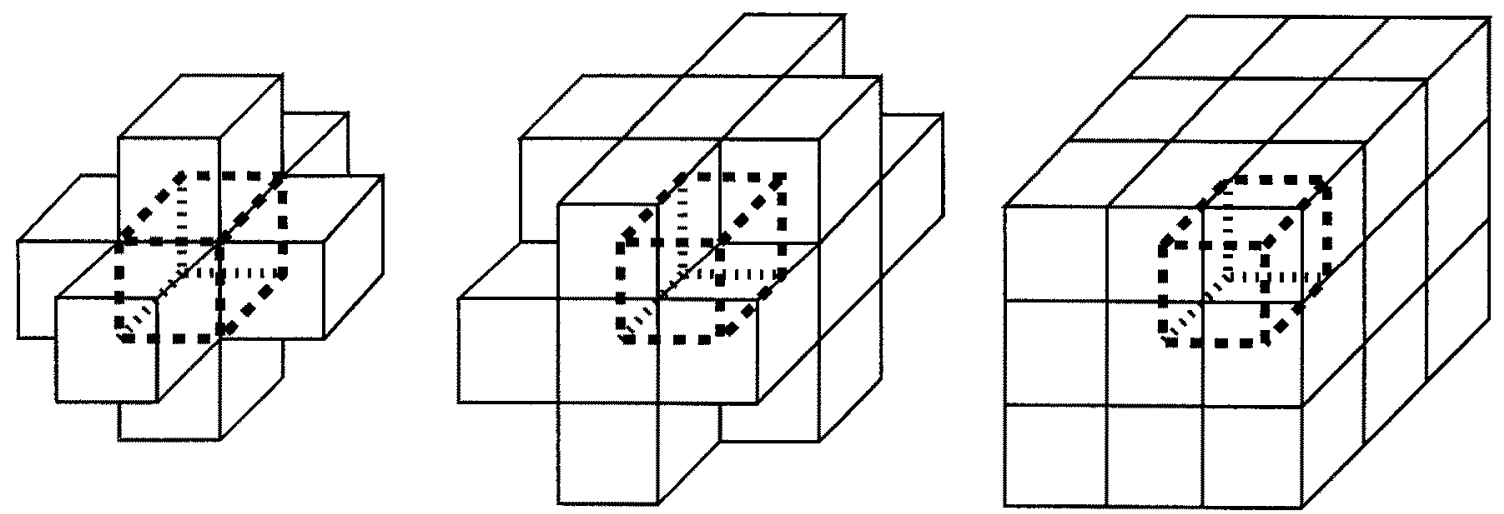

Fig. 5. The different neighborhoods in 3-D. From left to right: 6-neighborhood, 18-neighborhood, and 26-neighborhood.

their associated neighborhoods are defined by

$$
\begin{aligned}
& V_{1}^{i}(x)=\left\{y \in \mathbb{Z}^{3} / D_{1}(x, y) \leq i\right\} \\
& V_{\infty}^{i}(x)=\left\{y \in \mathbb{Z}^{3} / D_{\infty}(x, y) \leq i\right\} .
\end{aligned}
$$

We have then $N_{6}(x)=V_{1}^{1}(x), N_{26}(x)=V_{\infty}^{1}(x)$, and $N_{18}(x)=V_{1}^{2}(x) \cap V_{\infty}^{1}(x)$.

As in the 2-D case, different connectivities should be used in a binary image for the object $X$ and the background $\bar{X}$ in order to avoid any connectivity paradox.

\subsection{Other Definitions}

Let $n$ be in $\{6,18,26\}$.

Two subsets $X \subset \Sigma$ and $Y \subset \Sigma$ are said to be $n$ adjacent if there exists $x \in X$ and $y \in Y, x$ and $y$ being $n$-neighbors.

An $n$-path is a sequence $\rho_{0}, \rho_{1}, \ldots, \rho_{i}, \ldots, \rho_{k}$ of points such that $\rho_{i}$ is $n$-adjacent to $\rho_{i-1}$, for $1 \leq i \leq$ $k$. An object $X \subset \Sigma$ is $n$-connected if an $n$-path lying in $X$ can be found between each pair of points of $X$.

An $n$-connected component of $X$ is a set $Y \subset X$ which is $n$-connected and which is maximal for this property.

Let us introduce the notions of hole and cavity. The notion of hole is not simple to define-see (Park \& Rosenfeld 1971; Morgenthaler 1980). For example a torus has one hole. The notion of cavity is intuitive: for example a ball has one cavity, its interior.

The genus of $X, G(X)$ is the number of $X$-connected components plus the number of its cavities minus the number of its holes. $G_{n}(X)$ is the genus of $X$ computed with the $n$-connectivity.

If $X$ is finite, we have the following relations (Morgenthaler 1980):

$$
G_{26}(\bar{X})-G_{6}(X)=1, \quad G_{6}(\bar{X})-G_{26}(X)=1
$$

A method for obtaining the genus of a 3-D object by local pattern matching is given for the 6-connectivity case in Park and Rosenfeld (1971). Morgenthaler (1980) gives a method for the 26-connectivity case.

A point $x \in X$ is called simple if its removal does not change the topology of the image. Kong and Rosenfeld (1989) give a characterization of simple points which is an improvement of the one given by Morgenthaler (1981). A point $x$ is simple if

$$
\begin{aligned}
N C_{a}\left[X \cap N_{26}^{*}(x)\right] & =1 \\
N C_{a}\left[\bar{X} \cap N_{26}^{*}(x)\right] & =1 \\
G_{26}\left[X \cap N_{26}(x)\right] & =G_{26}\left[X \cap N_{26}^{*}(x)\right]
\end{aligned}
$$

where $N C_{a}(A)$ stands for the number of connected components of $A$ adjacent to $x$.

\section{Topological Classification in $\mathbb{Z}^{3}$}

Let $\Sigma$ be a 3-D binary digital image made of the object $X$ and the background $\bar{X}$. In the following, we assume that 26-connectivity is used for $X$ and 6-connectivity is used for $\bar{X}$.

In this section, we adapt the definitions of the real case (see section 2) to the discrete case. Then we discuss the choice of the neighborhood where numbers of connected components will be computed.

\subsection{Numbers of Connected Components}

We change the previous definition of the numbers of connected components for the discrete case. We define $C^{*}$ and $\bar{C}$ in a neighborhood $V$ of an object point $x$ by 
$C^{*}$ number of 26-connected components of $X \cap$ $V^{*} 26$-adjacent to $x$

$\bar{C}$ number of 6-connected components of $\bar{X} \cap V$ 6-adjacent to $x$

By using the same type of definition, the two other numbers of connected components, $C$ and $\bar{C}^{*}$, are

$C$ number of 26-connected components of $X \cap V$ 26-adjacent to $x$

$\bar{C}^{*}$ number of 6-connected components of $(\bar{X} \cap$ V) $\cup\{x\}$ 6-adjacent to $x$ ( $x$ is considered as a background point.)

By definition of $C$ and $\bar{C}^{*}$, we have $C=\bar{C}^{*}=1$ (proposition 1 is always verified).

\subsection{Neighborhoods}

Unlike the real case, we cannot choose an arbitrary small neighborhood $V$ in order to count connected components. We have only neighborhoods of fixed size.

If we consider the 26-connectivity, it is obvious that the smallest neighborhood we can choose for computing $C^{*}$ is the 26-neighborhood $N_{26}$. Because all points of the 26-neighborhood are 26-adjacent to the central point, it is not necessary to check the 26-adjacency to $x$ of the 26-connected components.

If we consider now the 6-connectivity, we can count the 6-connected components in the 26-neighborhood too-see (Malandain, Bertrand, \& Ayache 1991a; 1991b).

We have already seen (in section 3.1) that the 26-connectivity corresponds to the $D_{\infty}$ metric while the 6-connectivity corresponds to the $D_{1}$ metric. The 26-neighborhood is a sphere of radius 1 for the $D_{\infty}$ metric $\left(N_{26}(x)=V_{\infty}^{1}\right)$, and verifies the properties 1 and 2. The sphere of radius 1 for the $D_{1}$ metric is the 6-neighborhood $N_{6}$. But $N_{6}^{*}$ is not connected and thus the 6-neighborhood does not verify the properties 1 and 2. Let us consider now the sphere of radius 2 for the $D_{1}$ metric, $V_{1}^{2}(x)=\left\{y \in \mathbb{Z}^{3} / D_{1}(x, y) \leq 2\right\}$ (see figure 6). It verifies the properties 1 and 2 . It is an octahedron which differs from the 18-neighborhood by its six corners (since $N_{18}(x)=V_{1}^{2}(x) \cap V_{\infty}^{1}(x)$ ). Each of these six corners has only one 6-neighbor (which belongs to the 18-neighborhood); then it belongs to a 6-connected component 6-adjacent to $x$ if and only if this 6-neighbor belongs to the 6-connected component too. Thus, the number of 6-connected components 6-adjacent to $x$ is the same in the 18-neighborhood $N_{18}(x)$ and in $V_{1}^{2}(x)$. These six corners do not bring any additional topological information.

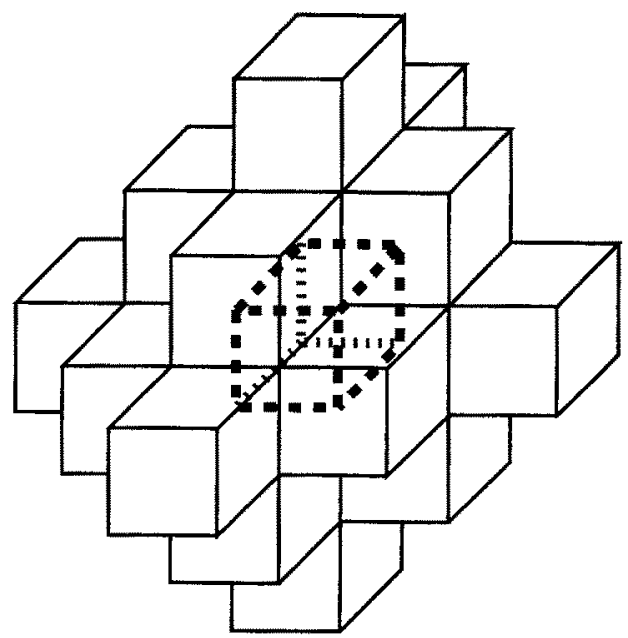

Fig. 6. The sphere of radius 2 for the $D_{1}$ metric $\left(V_{1}^{2}(x)\right)$ is an octahedron.

Because the 18-neighborhood is smaller than the 26-neighborhood, it will bring a more precise topological information-see (Malandain 1992; Bertrand \& Malandain 1992b).

\subsection{Conclusion}

Because of the different connectivities used for both the object and the background, we use different neighborhoods for counting numbers of connected components.

For each point of a 3-D binary digital image, we can apply the classification given in table 1 by using the following two numbers:

$C^{*}$ number of 26-connected components of $X \cap$ $N_{26}(x)$

$\bar{C}$ number of 6-connected components of $\bar{X} \cap$ $N_{18}(x)$ 6-adjacent to $x$

It should be pointed out that we assign a class to every possible value of $\bar{C}$ and $C^{*}$. It means that there will not remain any unclassified point, whereas this was the case previously (Morgenthaler \& Rosenfeld 1980).

These two numbers can be efficiently computed by using the a priori knowledge of the possible adjacencies in such neighboroods (Malandain 1992).

\section{Detection of Junctions}

After the topological classification presented in the previous section, each point of a 3-D binary digital 
image is labeled with its topological type (see table 1). However, some junctions can not be locally detected, because of the discretization of the space.

\subsection{Junctions}

Some troubles occur with the detection and the classification of junction points (between curves or surfaces) depending on their "thickness." The following figures (figures 7, 8,9), which represent a slice of a 3-D binary image, may be considered either as a junction between curves (with empty lower and upper slices) or as a junction between surfaces (with identical lower and upper slices). If we consider figure 7 , which represents a "thin" junction, the junction point is well classified by the local topological classification.

If we consider figure 8, which represents a "thick" junction, the junction point is misclassified: it is classified as a surface point if the figure represents a junction between curves, or as an interior point if the figure represents a junction between surfaces. In this case, the junction between curves is considered by the local classification as a little surface from which curves are emanating, and the junction between surfaces as a little volume (like a cylinder) from which surfaces are emanating.

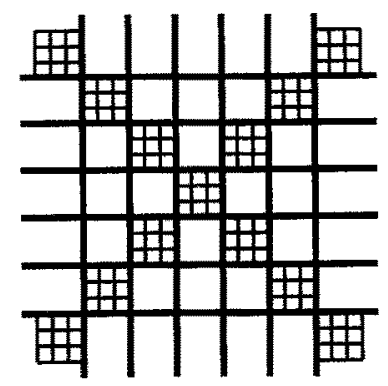

Fig. 7. The junction point is detected by the classification.

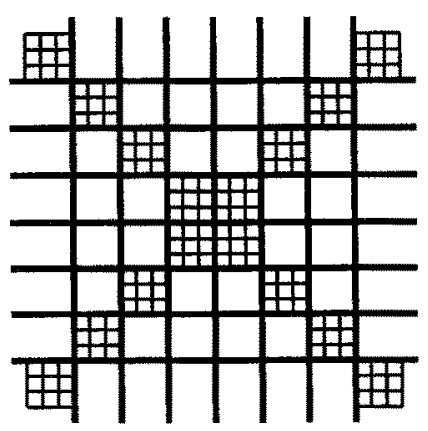

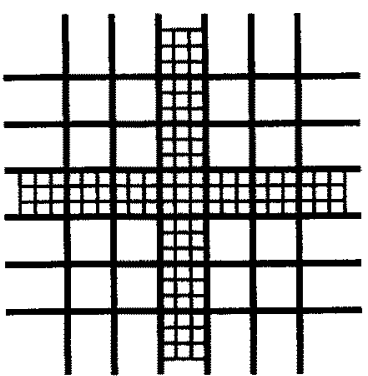

Fig. 8. The junction point is classified either as a surface point for curves junction, or as an interior point for surfaces junction.

Finally, if we consider figure 9 , which represents two other types of "thick" junctions, the junction points are undetected by the local classification: all points are classified either as curve points or as surface points.

\subsection{Curves Junctions}

The detection of the missed curves junction points can be easily made by counting for each curve point the number of neighbors that are curve points too. If this number is greater than two, then the curve point is a missed curves junction point.

\subsection{Surfaces Junctions}

The detection of the missed surfaces junction points is more difficult.

We call surface a connected set of points with a thickness of 1 (the deletion of any surface point will create a hole). A surface can consist of several simple surfaces linked together by junction (T-junctions, crossjunctions, ....).

As discussed above the connected components numbers do not allow, in a complex figure, to separate

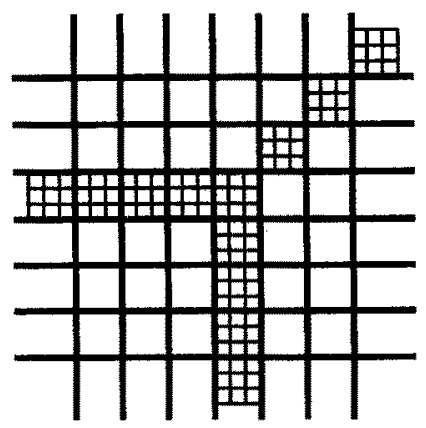

Fig. 9. All points are classified as curve points or as surface points. 
simple surfaces from each other (see figure 9 for example): if we delete all junction points of an image the remaining connected components are not necessarily simple surfaces.

Some work has been done previously to characterize simple surfaces (Morgenthaler \& Rosenfeld 1980; Kim 1984; Keskes \& Faugeras 1981). We consider the definition of a simple surface proposed in (Morgenthaler \& Rosenfeld 1980) because a simple surface is considered here as a set of voxels rather than a set of faces as in (Keskes \& Faugeras 1981). We first compare this definition with our classification, then we show that this definition is not appropriate, finally we propose an algorithm for the complete extraction of simple surfaces.

A point $x \in X$ is a simple surface point, according to Morgenthaler and Rosenfeld (1980), if the following conditions are all satisfied:

1. $X \cap N_{26}^{*}(x)$ has exactly one component adjacent to $x$ (in the $X$ sense).

2. $\bar{X} \cap N_{26}^{*}(x)$ has exactly two components adjacent to $x$ (in the $\bar{X}$ sense); call these components $B_{x}$ and $C_{x}$.

3. For every $y \in X$ adjacent to $x$ (in the $X$ sense), $y$ is adjacent (in the $\bar{X}$ sense) to some point in $B_{x}$ and to some point in $C_{x}$.

If we use 26-connectedness for $X$, we can see that condition 1 is equivalent to $C^{*}=1$, condition 2 is equivalent to $\bar{C}=2$ (but computed in the 26 -neighborhood) hence these two conditions characterize what we call surface points. Let us consider now figure 9: all points except the four (or the three) central one will be simple surface points. So this characterization succeeds in segmenting the complex surface into simple ones.

However we did not retain this definition because it is not always appropriate. In figure 10 one can see
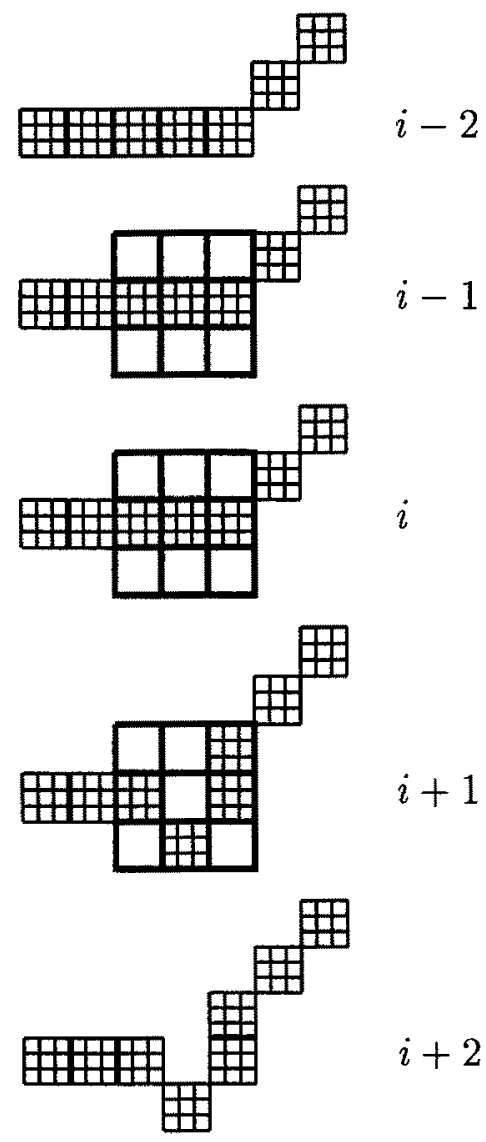

Fig. 10. The point at the center of the window of the $i$ th plane is not a simple surface point: the point at the upper right corner of the window in the $(i+1)$ th plane does not satisfy condition 3 . See figure 11 for a 3-D display of the surface.

a set of points which should normally be interpreted as a simple surface (see figure 11): the central point of the $i$ th plane is not a simple surface point according to the above definition.
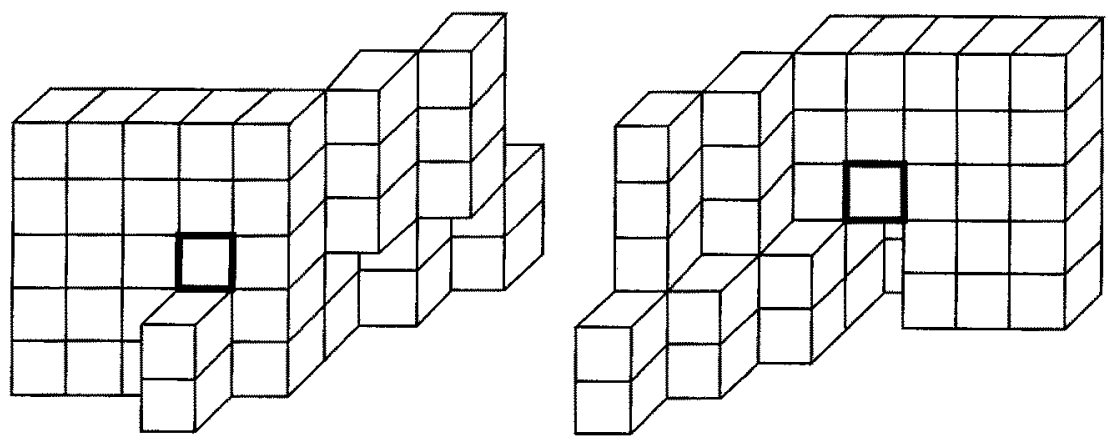

Fig. 11. 3-D display of the surface of figure 10 . 
To overcome this difficulty, we propose to use an approach based on an equivalence relation. The basic idea is to detect the simple surfaces defining a missed junction instead of the junction itself.

Let $x$ be a surface point. We call $B_{x}$ and $C_{x}$ the two connected components of $\bar{X} \cap N_{i}^{*}(x)$ 6-adjacent to $x$. We say that two surface points $x$ and $y$ are in relation if there is a 26-path $x_{0}, x_{1}, \ldots, x_{i}, \ldots, x_{n}$ with $x_{0}=$ $x$ and $x_{n}=y$ such that for $i \in[0, \ldots, n-1]$ :

$-B_{x_{i}} \cap B_{x_{i+1}} \neq \emptyset$ and $C_{x_{i}} \cap C_{x_{i+1}} \neq \emptyset$, or $-B_{x_{i}} \cap C_{x_{i+1}} \neq \emptyset$ and $C_{x_{i}} \cap B_{x_{i+1}} \neq \emptyset$

It is easy to check that this relation is reflexive, symmetric and transitive, that is, it is an equivalence relation. We call simple surface any equivalence class of this equivalence relation.

Using this equivalence relation, we are able to detect the undetected junctions. Let $x$ be a point of a simple surface $S \subset X$. If $x$ has a 26-neighbor $y$ which is a surface point and does not belong to $S$ but to another simple surface $S^{\prime}$, we say that $x$ is a junction point between $S$ and $S^{\prime}$.

A problem can still exist if a junction is generated by several parts of the same simple surface (see figure 12 where one simple surface generates a junction identical to the one of figure 9).

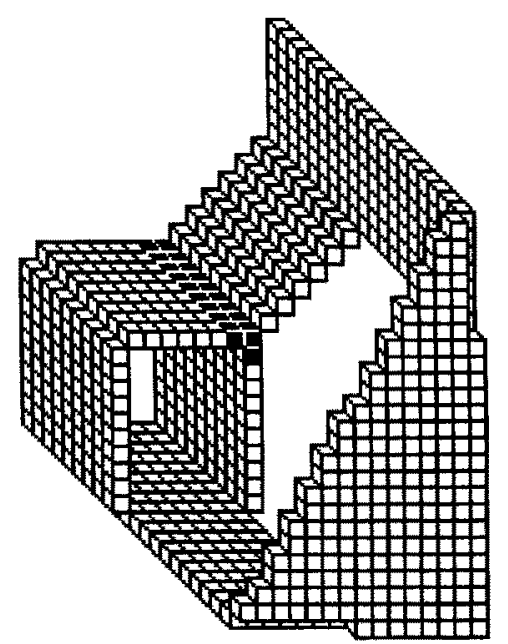

Fig. 12. Example of a single simple surface (in white) which generates a junction (in black).

Let us define $S(x)$ as the set of the surface points in $N_{26}(x)$. We say that a surface point $x$ is a junction point if $S(x)$ is not included in a single simple surface (according to the above definition). It is obvious that we must work in a $5 \times 5 \times 5$ neighborhood of $x$ for checking if $x$ is (or is not) a junction point.

\subsection{Conclusion}

By using the numbers $C^{*}$ and $\bar{C}$ we have obtained a first topological classification of each object point of a 3-D binary image. Since some junction points are undetected, we use a less local approach to detect them. After these two steps, we have a topological segmentation of a 3-D object into surfaces and surface junctions.

\section{Thinning and Topological Classification}

As we have seen, we have obtained a topological classification for each object point of a 3-D binary image. This classification provides us with a segmentation of this object in volumes, surfaces, curves, and junctions between surfaces and/or curves. Because these last two categories are more powerful for describing an object than volumes, the topological classification is then appropriate for complex objects made of surfaces and curves. These surfaces and curves have to be one pixel thick to be well classified (all points of a two-pixels thick surface will be classified as border points).

Objects that are more than one pixel thick can be found in edge images. In 3-D, edges are surfaces (because they separate volumes) which are rather thin if obtained by suppression of nonmaxima of the gradient or by extraction of the zero-crossings of the laplacian. However, it is safer to apply a thinning algorithm to these edges in order to be sure to have one-pixelthick surfaces.

Such objects may also be found in skeleton images obtained by applying a thinning algorithm until stability, There is two types of thinning algorithm: the first is designed for thinning surfaces, the second is designed for thinning curves. We obtain then a complex set of surfaces or a complex set of curves.

In both cases, thinning is an important step before the topological classification. Several algorithms have been proposed for thinning 3-D objects-see (Morgenthaler 1981; Lobregt, Verbeck, \& Groen 1980; Tsao \& Fu 1982; Hafford \& Preston 1984; Gong \& Bertrand 1990). Two kinds of methods have been considered: one reduces objects to surfaces and curves, the other transforms objects solely into curves. The basic idea is to remove in parallel simple points which satisfy some "geometrical" properties.

\subsection{New Characterization of Simple Points}

A point is called simple if its removal does not change the topology of the image. Kong and Rosenfeld (1989) 
present a characterization with three conditions (see section 3.2) using the genus-as mentioned in their article, it is essentially due to (Tsao \& Fu 1982).

According to our topological classification (see table 1), we get indirectly a new characterization of simple points which does not need the computation of the genus.

Proposition 2. A point $x$ is simple if and only if it verifies

$$
C^{*}=N C_{a}\left[X \cap N_{26}^{*}(x)\right]=1
$$

and

$$
\bar{C}=N C_{a}\left[\bar{X} \cap N_{18}(x)\right]=1
$$

The proof of this proposition may be found in Bertrand and Malandain (1992a).

\subsection{A Thinning Algorithm}

The above definition of simple points can be used for designing a new thinning algorithm. Instead of proposing such an algorithm. we prefer to refer to (Tsao \& Fu 1981), where thinning algorithms are characterized by two criteria: one of them can be easily modified for using the new definition of simple points.

\section{Detection of Significant Simple Surfaces}

Let us consider a complex object made of surfaces. If we delete all junction points, the remaining connected components are necessarily simple surfaces. These simple surfaces with their adjacencies (given by the junctions) constitute a topological description of the object.
However, some of these simple surfaces are not significant, because they are too small.

In order to detect only significant simple surfaces, we use a mathematical morphology approach-see Serra $(1982 ; 1988)$.

Given a set of simple surfaces, we "erode" them by suppressing their borders (the borders are detected by the topological classification). Be repeating this operation, we perform an erosion of order two, etc.

Then, we can either dilate the eroded simple surfaces into the original ones (the dilation has the same order as the erosion): we perform an opening of the simple surfaces; or reconstruct significant simple surfaces by dilating the eroded simple surfaces into the original ones until stability. In both cases, we obtain the significant simple surfaces of the original complex object.

An erosion of a great order requires topological classification of all points at each iteration. Then, in order to decrease the computational cost, it is better to implement the erosion of greater order by using a distance transformation-see Borgefors (1984)-followed by a thresholding.

\section{Experimental Results}

\subsection{Vertebrae}

We consider two $X$-Ray CT scanner 3-D images of a vertebra scanned in two different positions (see figures 13 and 14). They contain about $256 \times 256 \times 50$ voxels (each voxel is $0.5 * 0.5 * 1 \mathrm{~mm}^{3}$ ) whose intensity is coded with 256 discrete values.

The two images of vertebrae can be registered by the application of a geometric transformation which is the
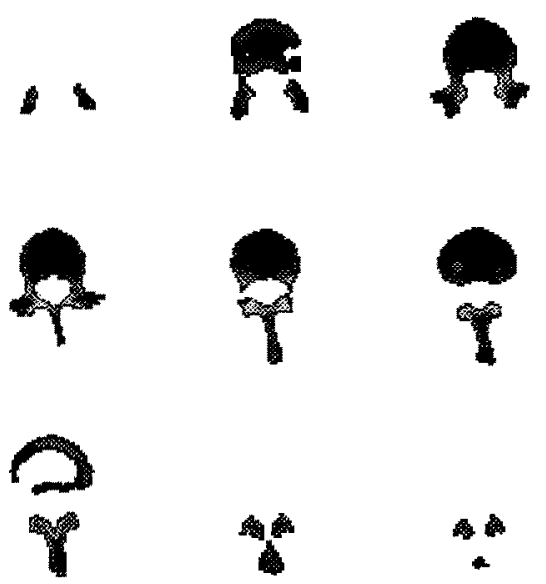
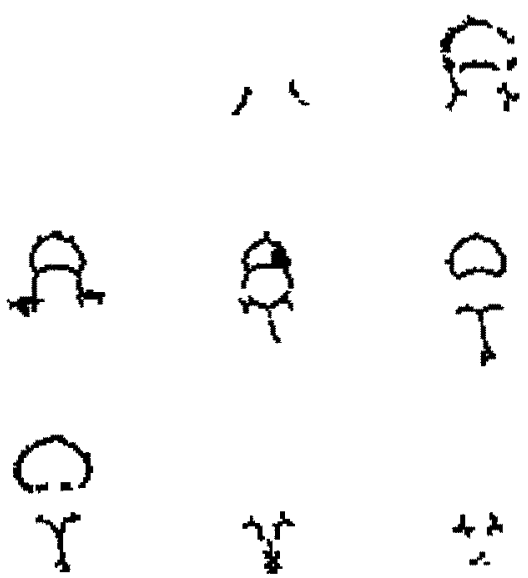

Fig. 13. A few slices of the vertebra scanned in the first position, and the corresponding skeleton. 

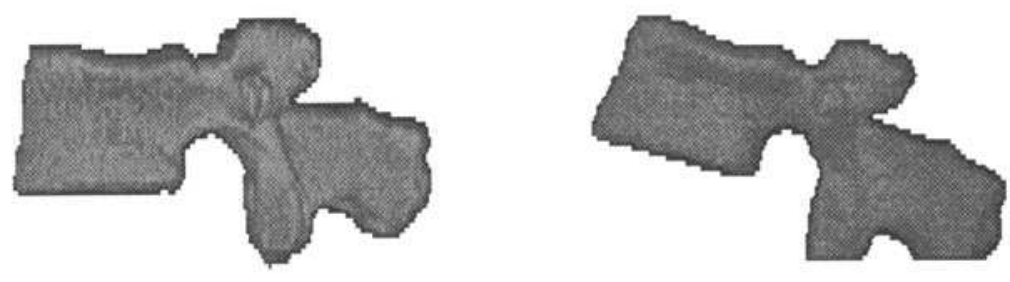

Fig. 14. A 3-D representation of vertebra in both positions.

combination of a translation, rotation and three different scalings along three orthogonal principal axes. This transformation was computed by matching globally the sets of data points: the rotation angle is 30 degrees along an axis that is tilted 11 degrees with respect to the vertical axis and scaling is close to 0.9 in two directions.

We applied the thinning algorithm to the entire vertebra as a 3-D object, producing a skeleton shown in figure 13. These skeletons each contain about 14,000 points, a figure which must be compared to the 170,000 original data points in each 3-D image. These skeletons, despite their instability to noise, are good examples for our method because of the complexity of the surfaces they are made of.

Then, we applied our classification and simplesurface extraction algorithm to label each skeleton point. This computation takes 40 seconds of CPU time on a DEC 5000. We show in figures 15 and 16 the main

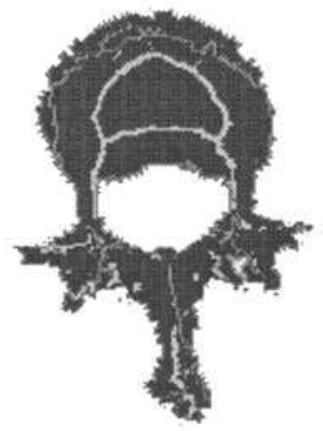

topological structures extracted for the two vertebrae. We extracted from these structures the junction points of both vertebrae, then we applied the geometric transformation found between them (see figures 17 and 18). It is easy to check the accurate superposition of the extracted structures between the two vertebrae. This shows the astonishing stability of the segmentation: a single quasi-rigid transformation can superimpose most of the structures within a tolerance of \pm 1 voxel.

Instead of junctions, one can use extracted simple surfaces. In order to obtain a robust segmentation of our surfaces, we perform an erosion of order 2 of the simple surfaces from their border toward their interior. We use for that computation a thresholding of the result of the distance transformation on simple surfaces. We apply our characterization and simple surface extraction algorithm to the result of erosion.

After this step of erosion, we apply a conditional dilation of order 2 of the remaining simple surfaces with

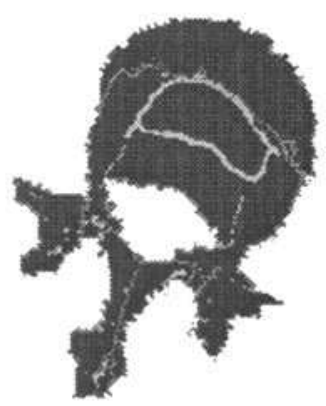

Fig. 15. Upper view of the skeleton of the vertebra in both positions. Surface points are dark grey, junction points are light grey, and edge points are medium grey.
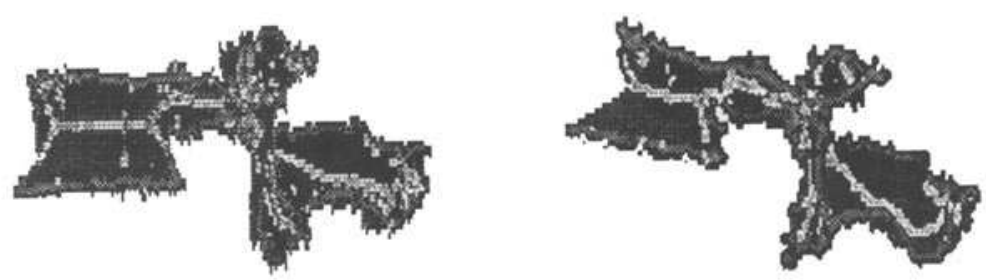

Fig. 16. 3-D representations of the skeleton of the vertebra in both positions (with rescaling in the third dimension). Surface points are dark grey, junction points are light grey and edge points are medium grey. 

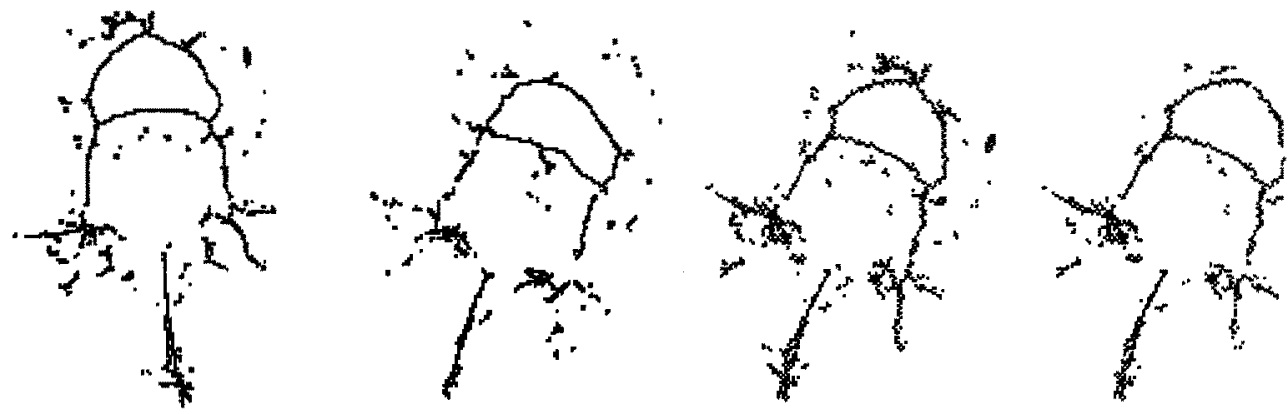

Fig. 17. Projections on the XY-plane of the junction points of the skeleton of (from the left to the right) the first vertebra (1), the second vertebra (2), the first vertebra after the computed transformation (3), and the intersection of the dilated set of junction points of the second vertebra with the set of junction points of the first vertebra after transformation (4).
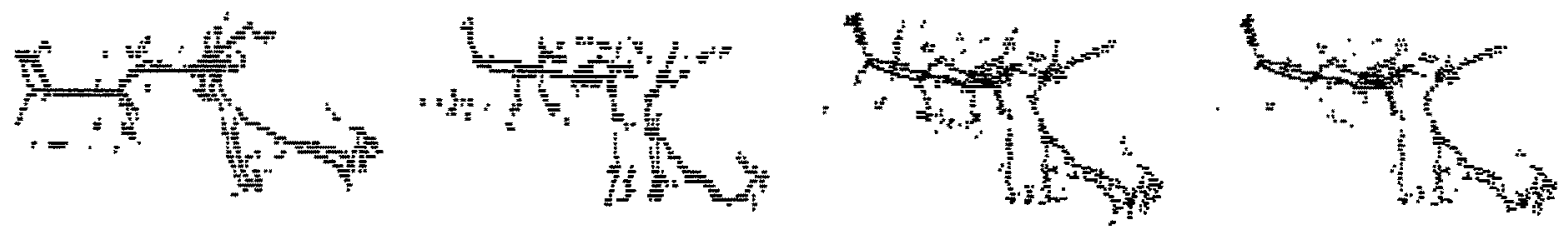

Fig. 18. Same as figure 12 with projections on the $Y Z$ plane.

respect to the original skeleton. This is an opening of simple surfaces. We extract the simple surfaces of this result and we label them. The result of the labeling step is shown in figures 19 and 20 . It is easy to check that we succeed in segmenting the complex surfaces into several simple surfaces which are stable (for more than $80 \%$ of them) from one object to the other. This can be used in a 3-D matching algorithm.

\subsection{Skulls}

Then, we consider two $X$-Ray CT scanner 3-D images of a skull scanned in two different positions (see figure 21 ). They are made of about 51 slices (each slice contains $512 \times 512$ pixels, each pixel is $0.4 \cdot 0.4 \mathrm{~mm}^{2}$ ), with a gap of $3 \mathrm{~mm}$ between two consecutive slices. As the vertebrae, both skulls can be registered by the application of a geometric transformation.
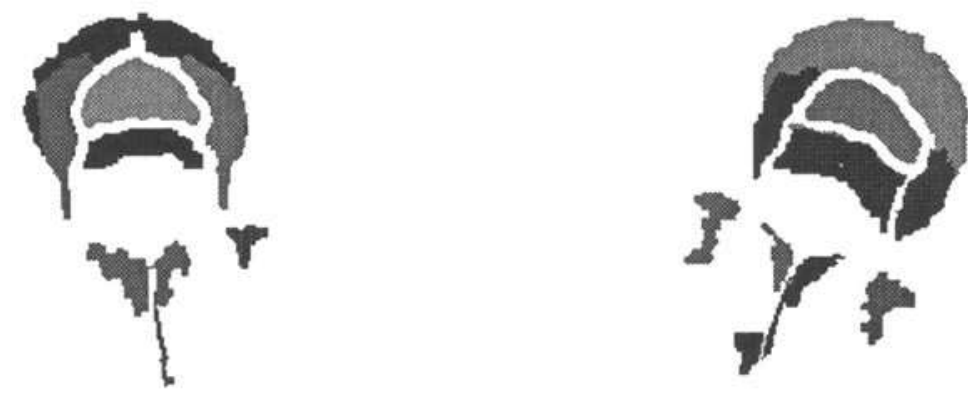

Fig. 19. Upper view of the skeleton of the vertebra in both positions after the labeling of simple surfaces remaining after an opening of order 2.
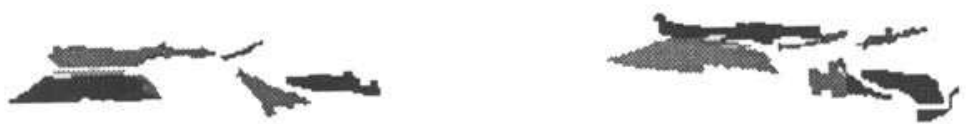

Fig. 20. Front view of the skeleton of the vertebra in both positions after the labeling of simple surfaces remaining after an opening of order 2 (without rescaling in the third dimension). 

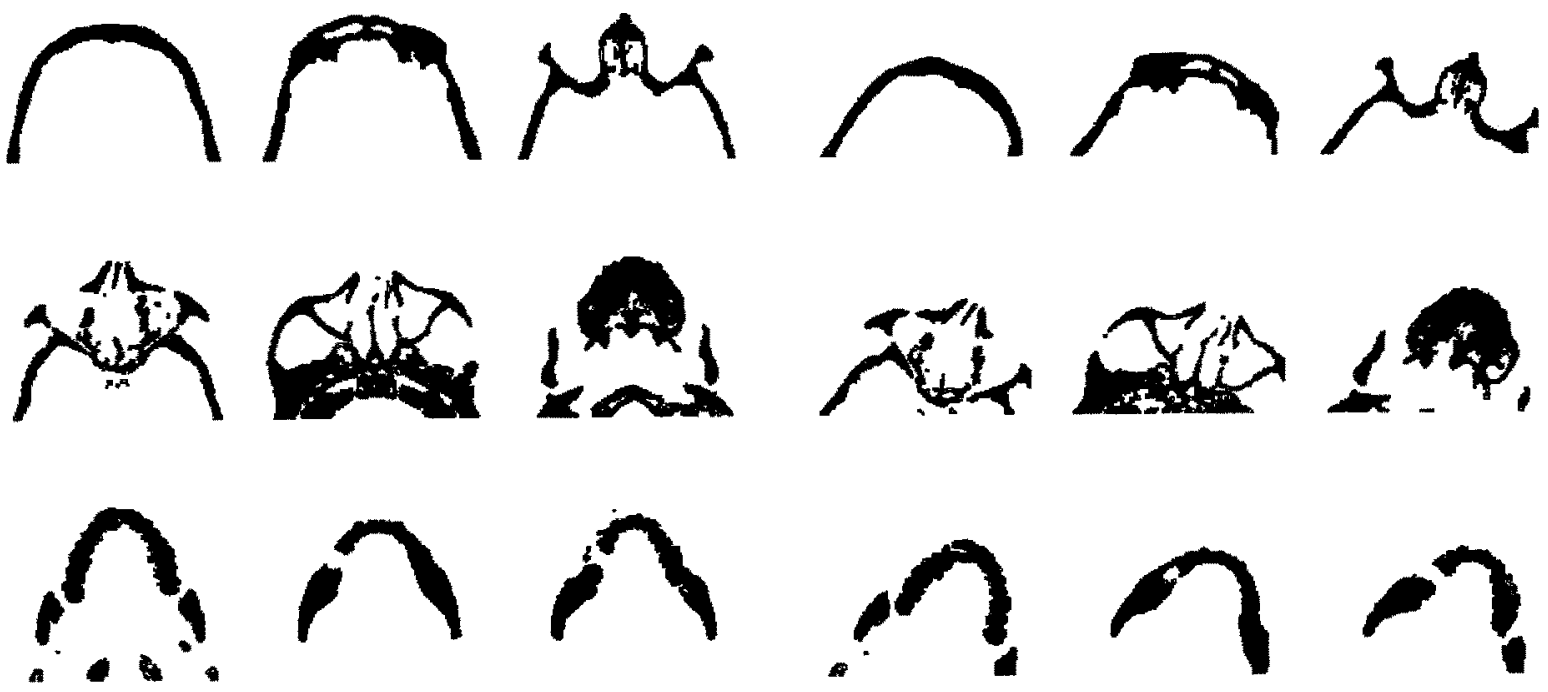

Fig. 21. A few slices ofthe skull scanned in the first position (to the left) and in the second position (to the right).

We interpolated the data to obtain quasi-isotropic voxels of $0.8 \cdot 0.8 \cdot 1 \mathrm{~mm}^{3}$, and we applied the thinning algorithm (see figures 22 and 23). We applied our classification algorithm to these objects to label each point. We shown in figure 24 the main topological structures extracted of the two skulls. They demonstrate, perhaps better than the vertebra example, the stability of the topological segmentation.

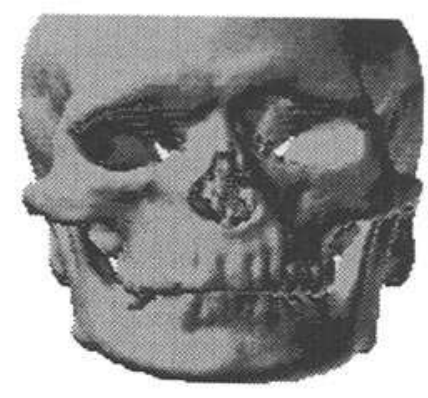

Fig. 22. A 3-D representation of skull in both positions.

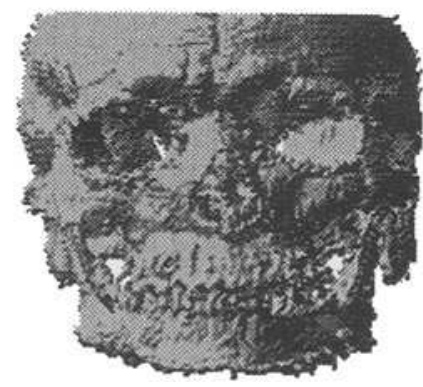

Fig. 23. A 3-D representation of a skull in both positions after thinning.

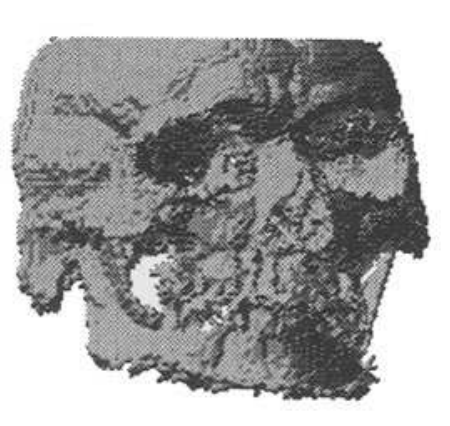

\section{Conclusion}

We have proposed a new approach to segment a discrete 3-D object into different classes of points which represent structures. The basic idea of this segmentation is the use of two local measures based on very local computations which allow massively parallel implementation. We obtain therefore a very fast segmentation of 3-D objects.

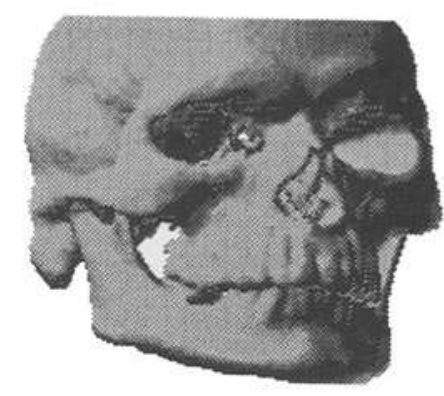



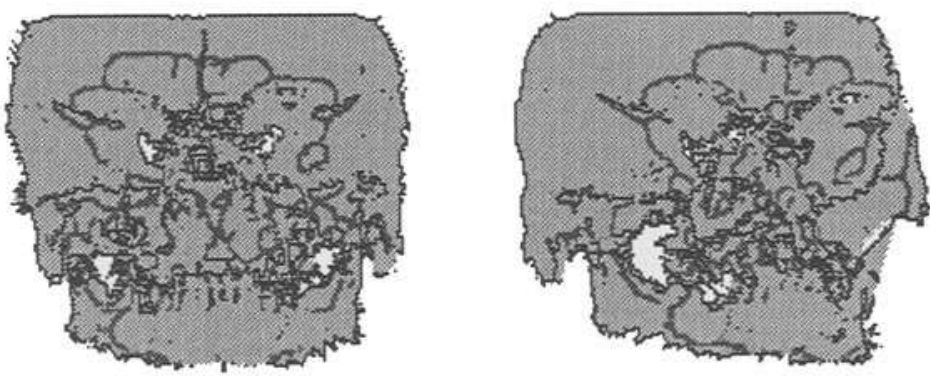

Fig. 24. 3-D-representation of the labeled skulls in both positions. Surface points are light grey, junction points are grey, and edge points are black.

The proposed classification gives a set of structures, junctions, or simple surfaces, which allows efficient registration of complex 3-D objects.

We applied our method for the segmentation into simple surfaces of the 3-D skeleton of a complex 3-D object, and the result appears to be remarkably accurate and stable with respect to quasi-rigid transformation, despite the relative instability of the skeleton itself.

Our method can be applied to the segmentation of 3-D edges, provided that the edge detector preserves the topology of junctions, which is not the case for known 3-D edge detectors.

Our future work will include a new 3-D edge detector, and the application of our segmentation method to 3-D edges, as well as its use to demonstrate robust matching of quasi-rigid complex 3-D objects.

\section{Acknowledgments}

This work was partially supported by Digital Equipment Corporation and European AIM (Advanced Informatics in Medicine) Project Murim. The authors want to thank Olivier Monga who initialized this research, and Pr. J.L. Coatrieux (Rennes Hospital, France) which provided us with the medical images of the vertebra. The images of the skull were acquired by the Advanced Image Processing Group of GE-CGR in Buc, France. Dr. Olivier Faugeras gave substantial help in the redaction of this article.

\section{References}

Ayache, N.; Boissonnat, J.D., Brunet, E., Cohen, L., Chièze, J.P., Geiger, B., Monga, O., Rocchisani, J.M., and Sander, P., 1989. Building highly structured volume representations in 3D medical images, Computer Aided Radiology, Berlin.
Ayache, N., Boissonnat, J.D., Cohen, L., Geiger, B., Lévy-Véhel, J., Monga, O. and Sander, P., 1990. Steps toward the automatic interpretation of $3 \mathrm{~d}$ images, Workshop on $3 \mathrm{D}$ Imaging in Medecine, Travemunde, R.F.A., June, NATO. Edited by Springer.

Bertrand, G., and Malandain, G., 1992a. A new characterization of three-dimensional simple points, Internal report, ESIEE, submitted for publication.

Bertrand, G., and Malandain, G., 1992b. A new topological segmentation of discrete surfaces, Proc. 2nd Europ. Conf. Comput. Vis. pp. 710-714, May 18-23, Santa Margherita, Ligure, Italy.

Borgefors, G., 1984. Distance transformations in arbitrary dimensions, Comput. Vis. Graph. Image Process. 27:321-345.

Gong, W.X., and Bertrand, G., 1990. A simple paralled 3D thinning algorithm, 10th Intern. Conf. Patt. Recog. June 17-21, Atlantic City.

Hafford, K.J., and Preston, K., Jr., 1984. Three dimensional skeletonization of elongated solids, Comput. Vis. Graph. Image Process. 27:78-91.

Keskes, N., and Faugeras, O., 1981. Surface simple dans $z^{3}$, 3éme Congrès Reconnaissance des Formes et d'Intelligence Artificielle, pp. 718-729, AFCET, September.

Kim, C.E., 1984. Three-dimensional digital planes, IEEE Trans. Patt. Anal. Mach. Intell. 6(5):639-645.

Kovalevsky, V.A. 1989. Finite topology as applied to image analysis, Comput. Vis. Graph. Image Process. 46:141-161.

Kong, T.Y., and Rosenfeld, A., 1989. Digital topology: introduction and survey. Comput. Vis. Graph. Image Process. 48:357-393.

Lobregt, S., Verbeck, P.W., and Groen, F.C., 1980. Threedimensional skeletonization: principle and algorithm, IEEE Trans. Pat. Anal. Mach. Intell. 2(1):75-77.

Malandain, G., and Bertrand, G., 1992. Fast characterization of 3d simple points, 11 th Intern. Conf. Patt. Recog. August 30-September 3, The Hague.

Malandain, G., Bertrand, G., and Ayache, N., 1991a. Topological classification in digital space. In 12th Intern. Conf. Informat. Process. Med. Imaging, Wye, Kent, England, July. Lecture notes in Computer Science 511, Springer-Verlag: Heidelberg.

Malandain, G., Bertrand, G., and Ayache, N., 1991b. Topological segmentation of discrete surfaces, Proc: IEEE Conf. Comput. Vis. Patt. Recog., June 3-6, Hawaii.

Monga, O., Deriche, R., Malandain, G., and Cocquerez, J.P., 1990. Recursive filtering and edge closing: two primary tools for 3D edge detection. Proc. Ist. Europ. Conf. Comput. Vis., Nice, France, April. Lecture notes in computer science 427, Springer Verlag: New York. 
Morgenthaler, D.G., 1980. Three-dimensional digital topology: the genus. Tech. Rpt. 980 , Computer Science Center, University of Maryland, College Park, MD 20742, November.

Morgenthaler, D.G., 1981. Three-dimensional simple points: serial erosion, parallel thinning, and skeletonization. Tech. Rpt. 1005 , Computer Science Center, University of Maryland, College Park, MD 20742, February.

Morgenthaler, D.G., and Rosenfeld, A., 1980. Surfaces in three-dimensional digital images. Tech. Rpt. 940, Computer Science Center, University of Maryland, College Park, MD 20742, September.

Nakamura, A., and Aizawa, K., 1985. On the recognition of properties of three-dimensional pictures, IEEE Trans. Patt. Anal. Mach. Intell. 7(6):708-713.

Park, C.M., and Rosenfeld, A., 1971. Connectivity and genus in three dimensions. Tech. Rpt. 156, Computer Science Center, University of Maryland, College Park, MD 20742, May.

Rosenfeld, A., 1980. Three-dimensional digital topology. Tech. Rpt. 936, Computer Science Center, University of Maryland, College Park, MD 20742, September.
Sander, P., 1989. Generic curvature features from 3D images. IEEE Trans. Syst. Man. Cybern., special issue on computer vision, November.

Serra, J., 1982. Image Analysis and Mathematical Morphology, vol. 1. Academic Press: San Diego, CA.

Serra, J., 1988. Image Analysis and Mathematical Morphology: Theoretical Advances, vol. 2. Academic Press: San Diego, CA.

Toriwaki, J.I., Yokoi, S., Yonekura, T., and Fukumura, T., 1982. Topological properties and topology-preserving transformation of a three-dimensional binary picture. Proc, oth Intern. Conf. Patt. Recog. pp. 414-419, October, Munich.

Tsao, Y.F., and Fu, K.S., 1981. A parallel thinning algorithm for 3D pictures, Comput. Graph. Image Process. 17:315-331.

Tsao, Y.F and Fu, K.S., 1982. A 3D parallel skeletonization thinning algorithm, Proc. IEEE Conf. Patt. Recog. Image Process. pp. $678-683$. 